\title{
ANGPTL3: a therapeutic target for atherosclerosis
}

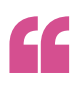

These studies

provide a

rationale for

pursuing the

development

of an

ANGPTL3

therapeutic

agent
Genetic and preliminary intervention studies published in two papers in NEJM indicate that angiopoietinrelated protein 3 (ANGPTL3) is a promising therapeutic target in patients with dyslipidaemia who are at risk of atherosclerotic cardiovascular disease. Together, the papers show that naturally occurring loss-offunction variants in ANGPTL3 are associated with reduced serum lipid levels and a lower risk of coronary artery disease, and that ANGPTL3 can be safely and effectively inhibited with the use of monoclonal antibodies or antisense oligonucleotides.

Angiopoietin-related proteins are important regulators of lipoprotein metabolism. ANGPTL3 is an endogenous inhibitor of lipoprotein lipase (LPL), which is the main enzyme involved in hydrolysis of triglyceride-rich lipoproteins.

Frederick Dewey and colleagues sequenced the ANGPTL3 exons in 58,335 participants of European ancestry in the DiscovEHR human genetics study. They identified 13 distinct loss-of-function variants in ANGPTL3. Overall, 246 individuals were heterozygous for loss-offunction variants, corresponding to a total carrier frequency of 1 in 237 . In 45,226

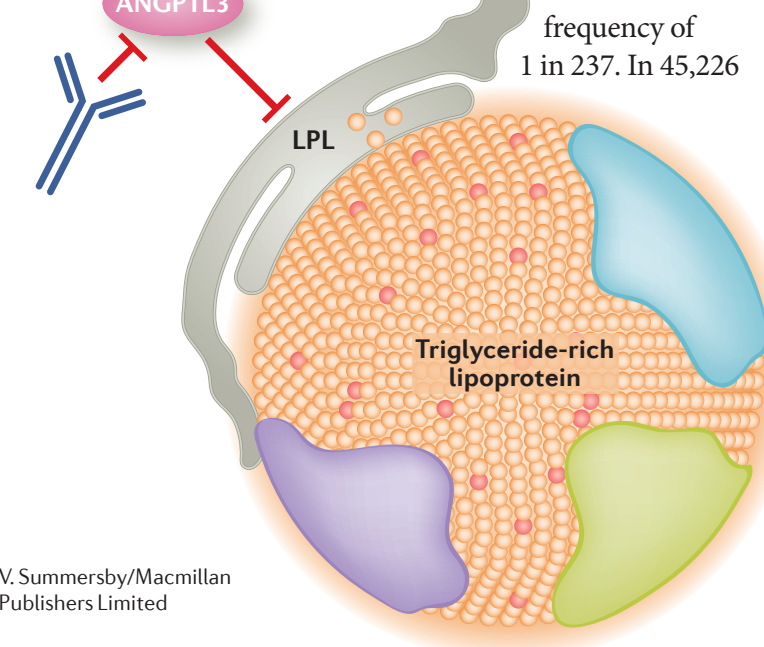

individuals with documented serum lipid levels, carriers of a loss-offunction ANGPTL3 variant had 27\% lower triglyceride levels, $9 \%$ lower LDL-cholesterol levels, and $4 \%$ lower HDL-cholesterol levels than noncarriers. In 13,102 patients with coronary artery disease and 40,430 controls, the presence of a loss-of-function variant was associated with a $41 \%$ lower risk of coronary artery disease (adjusted OR $0.59,95 \%$ CI $0.41-0.85, P=0.004)$.

The investigators next used $\mathrm{APOE}^{\star} 3$ Leiden.CETP mice, an established model of hyperlipidaemia, to investigate the effects of a fullyhuman anti-ANGPTL3 monoclonal antibody, evinacumab (REGN1500). Evinacumab administration was associated with significant reductions in total cholesterol level $(-52 \%)$ and triglyceride level $(-84 \%)$ compared with a control antibody. Evinacumab was also associated with a significantly greater decrease in atherosclerotic lesion size and necrotic content in the aortic root, but no significant difference in macrophage content, collagen content, or smooth muscle cell area.

Finally, Dewey et al. tested evinacumab in 83 healthy human volunteers with mildly elevated levels of triglycerides or LDL cholesterol. Participants were randomly assigned to subcutaneous or intravenous administration of evinacumab or placebo. The maximal changes in lipid levels were found in individuals who received a $20 \mathrm{mg} / \mathrm{kg}$ intravenous dose of evinacumab; triglyceride levels were reduced by $76.0 \%$, directly measured LDL-cholesterol levels by $23.2 \%$, and HDL-cholesterol levels by $18.4 \%$.

In a separate study, Mark Graham and colleagues tested antisense oligonucleotides targeting hepatic ANGPTL 3 mRNA in mice and humans. A mouse Angptl3 antisense oligonucleotide was administered to wild-type C57BL/6 mice, Lldlr-- mice, $A p o c 3^{-/-}$mice, and mice engineered to overexpress human apolipoprotein C-III. After administration of the antisense oligonucleotide, hepatic Angptl3 mRNA expression and plasma ANGPTL3 protein levels decreased in all mouse models. Administration was associated with reductions in serum triglyceride and LDL-cholesterol levels, as well as reductions in liver triglyceride content and atherosclerosis progression.

Next, 44 human participants with elevated serum triglyceride levels were randomly assigned to receive subcutaneous injections of placebo or an antisense oligonucleotide targeting ANGPTL3 mRNA in a single or multiple doses. After 6 weeks of treatment, participants in the multiple-dose groups had reductions in serum levels of ANGPTL3 protein, triglycerides, LDL cholesterol, VLDL cholesterol, non-HDL cholesterol, apolipoprotein B, and apolipoprotein C-III. No serious adverse events were reported.

"These studies provide a rationale for pursuing the development of an ANGPTL3 therapeutic agent as treatment for elevated levels of triglyceride-rich lipoproteins, in order to further reduce the risk of cardiovascular disease in persons who are already taking recommended medical and preventive therapies," conclude Graham and colleagues.

Gregory B. Lim

ORIGINAL ARTICLES Dewey, F. E. et al. Genetic and pharmacologic inactivation of ANGPTL3 and cardiovascular disease. N. Engl.J. Med.http://dx.doi.org/10.1056/NEIMoa1612790 (2017) | Graham, M. J. et al. Cardiovascular and metabolic effects of ANGPTL3 antisense oligonucleotides. N. Engl.J. Med.http://dx.doi. org/10.1056/NEJMoa1701329 (2017) FURTHER READING Reiner, Ž. et al. Hypertriglyceridaemia and risk of coronary artery disease. Nat. Rev. Cardiol. http://dx.doi. org/10.1038/nrcardio.2017.31 (2017) 